\title{
Percepciones sobre la degradación ambiental de agricultores orgánicos y convencionales en el ejido La Ciénega, municipio de EI Limón, Jalisco, México
}

\author{
Rodolfo GonZÁlEZ-FigueroA* \\ Peter R.W. GerRitSEN* \\ THerese K. MalischKe**
}

\begin{abstract}
Modern agriculture in Mexico is a cause of pollution and erosion among other problems. This results in an increasing poverty and marginalisation in many rural zones in the country, where the people's culture and sustain depend on agriculture. To understand and respond in the appropriate manner to the phenomenon of environmental degradation, we have to understand the ideas, values and hopes of those involved and based on this information come up with alternatives. In this paper we present four case studies in the ejido de la Ciénega, El Limón municipality, in the South Coast of Jalisco, Mexico and we contextualise these cases in the debate about sustainable development.
\end{abstract}

Keywords: perceptions, organic farming, agroindustrial agriculture, rural sustainable development.

\section{Resumen}

La agricultura moderna en México está causando contaminación y erosión; de ello deriva la creciente pobreza y marginación que se padece en diversas zonas rurales, donde la cultura y el sustento dependen de la agricultura. Entender y responder al fenómeno de la degradación ambiental implica comprender las ideas, valores y esperanzas de los campesinos y, a partir de ello, construir alternativas. En este artículo se presentan cuatro estudios de caso en el ejido de La Ciénega, municipio El Limón, en la región Costa Sur de Jalisco, México, buscando contextualizar estos casos en el debate sobre el desarrollo sustentable.

Palabras clave: percepciones, agricultura orgánica, agricultura agroindustrial, desarrollo rural sustentable.

* Universidad de Guadalajara, México. Correo-e: rodorganico@hotmail.com, petergerritsen@cucsur.udg.mx.

**Universidad de Wisconsin, Madison. Correo-e: tkmalichke@ gmail.com 


\section{Introducción $^{1}$}

La vida de los seres humanos depende de los recursos naturales de la tierra, desafortunadamente, una consecuencia de las tendencias actuales en la agricultura convencional es la degradación paulatina de los mismos. El término degradación tiene diferentes definiciones. En este artículo nos referimos a la degradación ambiental como una interrupción en los ciclos ecológicos y bioquímicos de restauración natural y a la explotación de recursos naturales a un ritmo tan rápido que éstos no pueden ser reemplazados. Las prácticas de monocultivo, el uso de agroquímicos, la manipulación genética, el riego y la labranza intensivos, contribuyen a un desequilibrio en los procesos naturales (Gliessman, 2000). Los agroquímicos contaminan el suelo y el agua, la materia orgánica se escurre de la tierra erosionando el suelo al tiempo que la diversidad de flora y fauna disminuye. La tendencia de la agricultura convencional también tiene efectos sociales. La forma como se practica esta agricultura conlleva una dependencia de insumos externos: tecnología, químicos y combustibles fósiles, además de que desplaza el poder de toma de decisiones de los campesinos a las empresas (frecuentemente transnacionales) que producen estos insumos (Toledo, 1995; Van der Ploeg, 1999; Jansen y Vellema, 2004).

Por lo aquí descrito, hoy en día podemos ver que "[...] la agricultura moderna es insostenible, a largo plazo no tiene el potencial para producir suficiente alimento como demanda la población debido, precisamente, a que está erosionando las condiciones que la hacen posible" (Gliessman, 2000: 3). El mismo autor también menciona que, para mantener un sistema agrícola sustentable, tenemos que basarnos en los ciclos ecológicos naturales y apoyar el desarrollo desde la base campesina.

En México, es urgente resolver estos problemas. En su libro México profundo, Guillermo Bonfil habla de la pobreza económica de un país con una gran riqueza en recursos y cultura:

Hoy debemos aceptar que México es un país pobre. Que grandes extensiones de tierra no son aptas para un cultivo moderno y que otras se han erosionado y producen menos porque se explotaron de manera irracional. Esto ha ido al extremo, de tal modo que nuestra agricultura no cosecha los suficientes productos básicos que

${ }^{1}$ Este artículo es una versión más elaborada del extenso (Malischke et al., 2005) que fue presentado en el v Congreso de la Asociación Mexicana de Estudios Rurales (AMER), en la ciudad de Oaxaca, Oax., los días 25-28 de mayo de 2005. 
se requieren para alimentar a los mexicanos siquiera en el nivel mínimo indispensable. Crece nuestra dependencia por hambre: el país en que se creó el maíz importa ahora maíz” (1994: 217).

En este mismo sentido, Morales agrega: "Ahora el campo mexicano, raíz y origen del México profundo, y ejemplo de diversidad ecológica y cultural está en grave riesgo. El sector rural de México pasa por uno de los periodos más difíciles, a causa de las políticas de desarrollo implementadas y que han ocasionado una seria crisis que amenaza la existencia de los campesinos, de los indígenas y sus familias, de los recursos naturales y de su cultura" (2003: 33-34).

De acuerdo con estos autores, la agricultura moderna (o convencional) no proporciona suficientes alimentos para la población mexicana. Al contrario, como mencionamos, este tipo de agricultura ha causado una fuerte dependencia hacia los insumos y el mercado. Por ejemplo, como una de las consecuencias del Tratado de Libre Comercio (TLCAN) con América del Norte, la producción barata de maíz de grandes empresas de los Estados Unidos fomenta la importación a su país de origen. La importación de este grano, un alimento que es la base de la cultura mexicana, amplía esta amenaza (Swentesius et al., 2003).

\section{Planteamiento del problema}

No se pueden investigar temas ambientales sin tomar en cuenta cuestiones humanas, y viceversa, ya que la vida de los seres humanos está interconectada con el medio ambiente y con los recursos naturales. Por tanto, abordar los problemas de degradación ambiental también requiere enfocar los problemas a los que se enfrentan los seres humanos.

Para revertir las tendencias actuales de la degradación y los métodos no sustentables, primero es necesario entender las percepciones de los campesinos ya que son ellos quienes toman las decisiones y trabajan la tierra. En su libro sobre percepciones indígenas, Lazos y Paré enfatizan que "La forma de intervenir en la realidad responde a la manera de percibirla. La integración, discusión y confrontación de los diferentes elementos queda como tarea primordial para hilvanar en torno a un desarrollo participativo y sustentable" (2000: 14). En otras palabras, sólo se pueden crear cambios profundos y duraderos para una mejor calidad de vida cuando la prioridad principal son las necesidades, creencias y valores de la gente directamente afectada por este cambio. La única manera de entender cuál es una mejor calidad 
de vida para la gente involucrada y qué resultados esperan, es entender sus percepciones. En este contexto, percepción se puede definir como: "La respuesta sensorial a los estímulos externos y a la actividad en la que ciertos fenómenos quedan registrados mientras otros quedan en la oscuridad. [...] El mundo como experiencia conceptualizada" (ibidem: 18).

Este artículo pretende describir algunos de los fenómenos de la agricultura desde conceptualizaciones campesinas, con base en algunos estudios de caso. Todas las cuestiones aquí discutidas, degradación, calidad de vida y creencias sobre la tierra, son ideas conceptualizadas, que cada persona filtra por su conocimiento predispuesto. A continuación describimos el diseño y área de estudio, para posteriormente presentar nuestros resultados. Terminamos con una discusión y conclusión.

\section{Diseño del estudio}

El objetivo de este artículo es describir las percepciones de algunos campesinos en torno a la degradación ambiental y sus implicaciones en los métodos de cultivo, haciendo una comparación entre productores orgánicos y convencionales. Las preguntas de investigación se dirigieron a entender los tipos de degradación ambiental que perciben los campesinos y el efecto de ésta sobre su calidad de vida. También analizamos las implicaciones de estas percepciones en esquemas que buscan reforzar el desarrollo regional sustentable.

El enfoque teórico aplicado es la perspectiva orientada hacia los actores sociales, desarrollado por Norman Long y sus colegas de la Universidad de Wageningen, Países Bajos (Long, 2001; Long y Long, 1992; Long y Van der Ploeg, 1989, 1994). Este enfoque parte de dos ideas básicas: supone que los actores sociales tienen conocimiento de su entorno social y natural y que cuentan con las habilidades y capacidades para transformarlo.

Durante el trabajo de campo, septiembre-diciembre de 2004, optamos por una investigación basada en estudios de caso ya que permite un mayor acercamiento al tema de nuestro interés. Los criterios de selección de los casos fueron la representatividad de los productores por su forma de trabajar en el ejido La Ciénega y su disponibilidad para participar en el estudio. La metodología consistió en entrevistas exhaustivas con cuatro campesinos -dos trabajan con métodos orgánicos y los otros con métodos convencionales- para conocer la historia de su vida. Las entrevistas se centraron en la historia familiar y sus prácticas agropecuarias, 
con una explicación del trabajo y la organización de la finca, así como preguntas enfocadas en los objetivos de investigación.

\section{Área del estudio}

Nuestra investigación se realizó en el ejido La Ciénega, risueña, tranquila y pequeña población rural ubicada al suroeste del estado de Jalisco, dentro de la región geográfica Costa Sur (figura 1). La vida cotidiana en La Ciénega está muy ligada al campo, más específicamente a la agricultura, de la que depende la economía local. En la actualidad se encuentra en una situación crítica debido a los bajos precios de los productos, la disgregación de los sistemas tradicionales de producción y el deterioro de los suelos. Como consecuencia, muchas personas se ven en la necesidad de realizar actividades complementarias, ya sea como jornaleros, peones, albañiles, mecánicos, plomeros, etc. Otros son ganaderos a pequeña escala, crían animales de traspatio y recolectan fruta silvestre de temporada. Los cultivos más importantes, por la superficie sembrada: maíz, sorgo, melón, sandía, pepino, limón, calabaza, agave azul, chile, frijol, cacahuate y papayo (Gerritsen et al., 2005; González, 2006; Imecbio, 2000).

\section{Figura 1}

\section{Ubicación geográfica del ejido La Ciénega, municipio de El Limón}

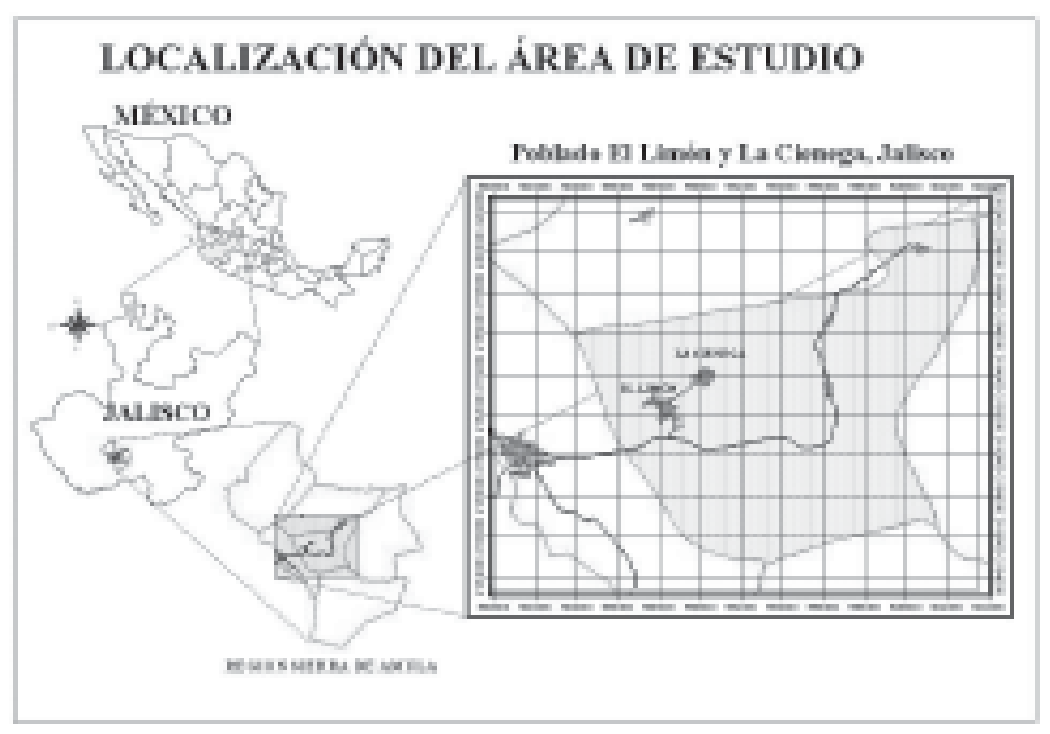


El ejido tiene una superficie de 1,183 hectáreas de terreno cultivable, 180 cuentan con riego, mientras que las restantes son de temporal. Ahora sólo están sembradas 606, como reflejo de la creciente migración, los efectos de una compañía agavera y las políticas de gobierno que tienden a descampezinar el campo.

\section{Resultados}

Como mencionamos, para entender mejor el fenómeno de la degradación ambiental es necesario escuchar directamente a los campesinos. ¿Qué percepciones tienen sobre el deterioro ambiental y qué efectos tienen en su vida? ¿Son diferentes las percepciones de campesinos que usan métodos orgánicos y aquéllos que utilizan agroquímicos? Con estas ideas y preguntas fuimos a La Ciénega para hablar con cuatro campesinos. A continuación presentamos de manera temática los resultados de las entrevistas.

\section{Los protagonistas}

¿Quiénes son los campesinos cuya plática e ideas forman la base de este artículo? Moncho (39 años), Raúl (47), José (40) y Osberto (36 años). ${ }^{2}$ Todos nacieron en La Ciénega, donde aprendieron a trabajar con sus padres y abuelos.

Moncho y Raúl trabajan juntos y están convencidos del método orgánico. Sin embargo, todavía usan fertilizantes químicos en algunos terrenos, mientras en otros fortalecen la tierra con abonos naturales y poco a poco avanzan dejando de usar fertilizantes químicos y tampoco aplican insecticidas ni herbicidas. Siembran maíz junto con calabaza, frijol y cacahuate; crían animales como cerdos, becerros, borregos, chivos y gallinas. José produce principalmente melón, aunque también siembra sorgo o maíz y engorda puercos y becerros; usa agroquímicos para fertilizar la tierra y controlar plagas; elimina las malezas manualmente con cultivadora o azadón, y en el peor de los casos, con herbicidas, aunque tiene interés en cambiar a métodos orgánicos. Osberto, quién solía sembrar melón, actualmente ya no lo hace por los bajos precios y la poca productividad. Ahora cultiva agave azul, sorgo y limón; usa agroquímicos pero por los altos costos de éstos se decidió sobre todo por los limones, ya que no ocupan mucho fertilizante y son más resistentes a las plagas.

${ }^{2}$ Por razones obvias, hemos cambiado los nombres de los entrevistados. 


\section{Una historia oral de La Ciénega}

Los agricultores entrevistados hablaron sobre la historia del ejido de La Ciénega, la cual comenzaron a narrar a partir del año 1922, en los inicios de la rebelión campesina por los pobladores y trabajadores en contra de los propietarios de las haciendas La Sidra y El Limón. Jacinto Galindo y un grupo de campesinos empezaron un movimiento para solicitar al gobierno estatal el reparto de tierras. Para finales de ese mismo año se comienza a formar el ejido La Ciénega, El Limón, así como el proceso de reparto de tierras en dicho ejido y una nueva etapa de desarrollo en el municipio.

Después de la conformación del ejido, los campesinos seguían sembrando maíz, chile y frijol. Se introdujeron programas y créditos gubernamentales para apoyar la producción del campo y asegurar la venta de las cosechas hasta Guadalajara: "No como ahora, que tienen que andar buscándole por todos lados”, dice uno de los productores ancianos del ejido.

Con la fundación del ejido, rápidamente se comenzó a poblar La Ciénega, pues había mucho terreno disponible para la agricultura. Se introdujeron nuevos cultivos, como cacahuate, calabazas, garbanzo y sorgo, entre otros. La mayoría se destinaba al mercado, mientras que la producción de frijol y maíz era para autoconsumo: "Dejábamos un poquito de maíz para nosotros y el frijol que poníamos era nomás pal gasto”, mencionó también.

En esos tiempos la principal actividad productiva era la agricultura, las técnicas que empleaban eran a base de la fuerza humana y tracción animal (caballos o bueyes), tenían herramientas de labranza fabricadas por ellos mismos, como el azadón y la casanga, mientras que los aperos para la yunta eran traídos de Guadalajara o Ciudad Guzmán. Prácticamente todas las familias criaban animales de traspatio: gallinas, guajolotes, cerdos, chivos, vacas, conejos, entre otros. Los alimentaban con forrajes verdes, zacates, restos de comida y algunos los sacaban a pastar al cerro. Parte de la alimentación familiar se basaba en fruta silvestre y otros productos que recolectaban del campo. "Por comida no sufríamos, siempre al salir al cerro o al potrero había cosas que comer, guarros, parotas, mesquites, quelites, verdolagas, guácimas [...] un chingadal [mucho]", nos comentó otro productor de avanzada edad. 


\section{La evolución de prácticas agrícolas}

En los años setenta del siglo xx, la Revolución Verde, una difusión de tecnología agrícola, empezó a cambiar los paradigmas sobre la agricultura en todas partes del mundo (Toledo, 2000). ¿Qué piensan los campesinos sobre los distintos cambios en la agricultura hasta las tendencias que vemos hoy? ¿Por qué piensan que las cosas cambiaron? José cree que la introducción de tecnología fue un fenómeno muy fuerte que cambió la cultura del campesino, y aunque al principio pareció un lujo no tener que trabajar tanto, ahora ve las consecuencias: ni con muchos insumos hay las mismas ganancias que antes.

Raúl habla de cómo la agricultura sigue evolucionando hacia el método de la tecnología, y de cómo estos cambios están afectando la cultura mexicana. Las recetas que ingenieros y agrónomos dan a los campesinos, supuestamente para mejorar sus cultivos, están reemplazando los conocimientos antiguos. En cuanto a la interacción entre la gente, cuando todos sembraban de manera tradicional, maíz, frijol y chile, hablaban el mismo idioma, todos eran agricultores, todos sembraban maíz. Ahora cada quien tiene su idioma, o sea, su punto de vista; la convivencia ya no es la misma.

Moncho comentó sobre la diversidad y autosuficiencia que tenían los agricultores en otro tiempo: gallinas, huevos, pollos para comer, maíz, frijol y tortillas hechas en casa. Mientras que en la actualidad, todo lo tienen que comprar.

\section{Lo orgánico vs. el sistema convencional: las diferencias}

Los científicos que están de acuerdo con la agricultura convencional dicen que la solución para incrementar la eficacia de la agricultura está en la implementación de tecnología e insumos. Una diferencia fundamental entre la agricultura convencional y la tradicional es que "El agricultor moderno puede aumentar en gran medida la producción en su área determinada usando los insumos adecuados, como fertilizantes, riego, drenaje, etc. De manera que, en su caso, la tierra pueda sustituirse en parte por la técnica y el capital" (Arnon, 1987: 60). Sin embargo, "para el agricultor tradicional, la tierra es el medio más importante para la producción y su única manera de supervivencia" (idem). Al hablar con estos cuatro campesinos, se evidencian diferencias agroecológicas, económicas y sociales. 
Todos los agricultores que entrevistamos tienen más o menos la misma superficie de terrenos, así como cultivos y productos. Quienes se dedican a los cultivos orgánicos hablaron más de la diversificación combinándolos con otros. Toda la familia trabaja y algunos emplean pocos jornaleros durante las épocas más pesadas.

Una gran diferencia entre los agricultores orgánicos y los convencionales es el destino de sus cultivos y cómo los comercializan. Raúl y Moncho están enfocados en producir primero para sus familias, y lo que sobra, para la misma comunidad. Osberto y José, por el contrario, con la ayuda de intermediarios venden sus productos en Guadalajara o incluso en Monterrey.

Otra diferencia es la forma de producir. Los agricultores convencionales, José y Osberto, utilizan un método menos complejo que involucra rastreo y surcada, escardas, fertilizantes, riego y aplicaciones químicas. Moncho, por su parte, explicó cómo él y Raúl, tratan de regresar a los métodos tradicionales. Con varios principios de la agricultura orgánica, utilizan herramientas como el azadón y la casanga para destruir el zacate sin necesidad de aplicar herbicidas, surcan la tierra con caballos, abonan con materia orgánica y conservan la semilla año tras año. Gastan menos dinero mediante el uso de materiales disponibles en vez de insumos externos, al tiempo que cuidan la tierra y preservan los cultivos tradicionales. En el manejo de residuos de cosecha y otros restos orgánicos, los agricultores convencionales queman la materia orgánica que queda en la parcela después de cosechar, mientras que los orgánicos la utilizan para nutrir la tierra.

\section{Degradación ambiental percibida por los campesinos}

Todos los campesinos con quienes hablamos perciben algunos tipos de degradación en su comunidad, como la que ocasionó el cultivo de agave azul, introducido en el ejido en 1997, el cual es altamente demandante de nutrientes del suelo y requiere aplicación intensiva de agroquímicos, lo que ocasiona la muerte de muchos árboles alrededor de las parcelas de agave, además de la degeneración del suelo y la materia orgánica. Aunque no usen pesticidas ni herbicidas, los agroquímicos que aplican otros agricultores afectan también sus cultivos. Otro efecto de los agroquímicos es la contaminación genética de las calabazas tradicionales con las de fuera, ya que ambas se empezaron a cruzar.

Un problema más es la degradación de la tierra en las parcelas rentadas, ya que los arrendatarios no le dan el mismo cuidado 
que los dueños y quedan dañadas, erosionadas y sin materia orgánica.

La cantidad y frecuencia de las lluvias también se mencionó. Los productores perciben que ya no llueve como antes, cambio que atribuyen a la tala de árboles. Los cultivos ya no son tan fuertes y en consecuencia ya no producen igual, problema que adjudican al cansancio de la tierra por sembrarla de manera intensiva.

Como última manifestación de la degradación ambiental los productores hablaron de los terrenos cansados, e intoxicados por la aplicación cada vez más fuerte de fertilizantes y agroquímicos. Ya no se produce con autosuficiencia porque la materia orgánica y los minerales intrínsecos han desaparecido a causa del mal manejo de los terrenos.

\section{Efectos de la degradación ambiental en la calidad de vida}

¿Cuáles son las preocupaciones de estos campesinos en relación con la degradación ambiental? Si la fertilidad de la tierra disminuye, es un problema grave para los productores, ya que de ella tienen su sustento. Éstos mencionaron una relación entre la contaminación de la tierra y su bienestar económico, y personal, así como en su salud.

José expresó que la "salud de sus terrenos" tiene un efecto económico para su familia, ya que si su tierra está dañada, necesita meterle más dinero para hacerla producir. Ante las enfermedades, José y Moncho creen lo que escuchan; de que tantos padecimientos en los niños son por el uso de agroquímicos. Moncho se preocupa por la clase de alimentos que está consumiendo su familia. Menciona que antes, sin la influencia de tantos químicos, la gente vivía más tiempo. Destacó que la salud de la tierra tiene un efecto directo en su bienestar personal: si la tierra está bien, él se siente bien; si está contaminada, se siente mal. Sólo uno de ellos, Osberto, ve que la degradación ambiental tiene un efecto para todos los agricultores, pero no menciona un efecto personal, sobre sus propios sentimientos y su bienestar. Para él, al parecer, es una correlación menos personal y menos espiritual.

\section{La relación de los campesinos con la tierra}

Todos los seres humanos tenemos una relación con la tierra, puesto que todos vivimos en ella. La concepción de Raúl y su unión con ella es que ahí trabaja, que de eso vive y de ahí viene su sustento. Para él, ser campesino es querer la tierra y sus organis- 
mos, son una fuente de paz, de consuelo, de tranquilidad. Raúl ve un valor en los procesos naturales y piensa que cada planta y cada organismo tienen su espacio en estos procesos, aun cuando algunos animales ocasionen daños a sus cultivos. La tierra no sólo le da un sustento físico, también le da un sustento espiritual y mental. No es nada más un trabajo, es su vida y él disfruta mucho de cada elemento de la misma.

Osberto piensa en sus terrenos como algo de subsistencia y mantenimiento, pero no comentó acerca de una relación personal más profunda. Habló sobre cuestiones económicas y de cómo no es rentable sembrar muchos cultivos por los altos precios de los químicos. Como resultado de la baja fertilidad de sus terrenos y lo caro de los insumos externos, ha perdido la fe de tener una calidad de vida buena basada en un trabajo diversificado y en conservar sus lazos con la agricultura. No ve razón en seguir con algo que no le va a dar ningún ingreso económico.

José tiene una relación cercana con su tierra por el conocimiento que tiene de la misma, pero admitió que tal relación se ha desvinculado un poco por practicar el modo de producción convencional. En cambio, la perspectiva de Moncho es de respeto hacia la tierra, así como de su cuidado y protección.

\section{Campesinos como actores en un entorno cambiante: limitaciones $y$ resistencias}

A medida que la agricultura moderna se fue introduciendo en el sistema de producción campesina, se transformó el pensar y el comportamiento tradicional. En la actualidad, los campesinos ya no actúan tanto con base en el conocimiento heredado, sino que la mayoría acude a asesoría técnica o se atienen a programas y apoyos gubernamentales (Toledo, 2000; Van der Ploeg, 1992, 1999).

Raúl ve que los campesinos mexicanos están perdiendo su soberanía, pues en lugar de adoptar una actitud ingeniosa, se dejan manejar por las ideas de técnicos y empresas que llegan de fuera. Enfatiza en la importancia del conocimiento de los campesinos y su propia iniciativa para resolver problemas. De la misma manera, Moncho comentó sobre la necesidad de los campesinos de ser activos en su aprendizaje y conocimiento. Por su parte, Raúl no ve limitaciones para seguir trabajando, desde su punto de vista, la escasez económica no es una limitante, porque de la tierra sale su subsistencia.

Con respecto a oportunidades de fuera, Raúl platicó acerca de la Red de Alternativas Sustentables Agropecuarias de Jalisco 
(RASA). Este grupo (no gubernamental) organiza reuniones entre los campesinos para que platiquen y compartan sus experiencias para seguir con métodos orgánicos; también ayuda a comercializar los productos ante la falta de interés del gobierno por avanzar con una agricultura sin químicos. ${ }^{3}$

Moncho comentó que además de no dar apoyos para agricultura orgánica, las acciones del gobierno en general están limitando más y más a los productores. También que es difícil interactuar con otros campesinos (convencionales) ya que sus percepciones son muy diferentes. Otra limitante para competir con otros productores son las ideas de los consumidores, porque, según él, son indiferentes ante la calidad de lo que consumen.

Osberto ve problemas al vender sus productos porque, mencionó, no hay una estabilidad y tampoco tiene confianza en enviar sus productos con compradores debido a que los precios son muy inestables y él gana muy poco, e incluso algunas veces, nada. Otro obstáculo es que los precios de los insumos son mucho más altos de lo que él gana al vender sus cosechas. Sin embargo, no ha pensado en cambiar a un sistema que no ocupa gastos de químicos. La solución que él ve es dedicarse más a plantar árboles, como los limoneros, que no ocupen mucho fertilizante.

José ve una gran limitación económica, por lo que está pensando en comenzar poco a poco con métodos orgánicos. Comentó que aunque la tecnología pareció un beneficio, al final ha terminado como un problema. Para él, cambiar sus métodos de cultivo no es algo que requiera ayuda de afuera o más conocimiento del que ya tiene, pues al respecto expresó que todavía conserva lo que aprendió de su papá. Reconoce su pasividad, y la de otros campesinos, en cuanto a que en lugar de fortalecer la tierra con su propio trabajo, se han ido por un camino en apariencia más fácil.

Ante los problemas que pueden surgir, Moncho enfatizó su principal estrategia para sobrevivir: resistencia, trabajo, echarle ganas y olvidarse de competir, usar la menor cantidad de químicos posible y buscar otras opciones siendo autosuficiente.

\section{Esperanzas para el futuro}

Para Raúl lo mejor es aprender más sobre lo que ya se está haciendo: la diversificación de cultivos y su combinación con la cría de animales, lo cual representa opciones sin tener que depender del

${ }^{3}$ Para mayor información sobre la RASA, véase Morales (2004). 
gobierno y de los precios, y que se enmarcan en las capacidades endógenas de seguir criando y produciendo. Para Osberto, sus esperanzas están puestas en cambiar al cultivo de árboles frutales que producen durante 20 años y así no tener que gastar tanto en químicos. En el caso de José, respondió que quiere empezar otra vez con los métodos orgánicos que antes usó su familia. Moncho opinó que la agricultura orgánica es una de las soluciones que tendrán que seguirse, aunque implique mucho trabajo.

A pesar de que sus métodos actuales son diferentes, todos los productores entrevistados ven que ahora la tierra está cansada, que no da como antes y que hay que buscar cambios en la forma de sembrar para continuar con su manera de vivir como agricultores. Si es iniciar con árboles frutales que no ocupan tantos químicos, es necesario incorporar materia orgánica en el terreno o seguir con lo que ya se está haciendo con métodos orgánicos. Todos reconocen que sus terrenos no pueden seguir produciendo con las promesas de la agricultura moderna. Moncho comentó:

Yo pienso que va a llegar el día en que un poco ya la gente esté viendo lo que está mal, y lo que está bueno. Porque ya se habla de lo orgánico. Y pienso que ya viendo un cambio de esto, a lo orgánico, pienso que va a ser un beneficio para nosotros mismos y vamos a estar un poco más avanzados que los demás que apenas van a querer iniciar [...] Ya tenemos tierras bien abonadas, yo pienso. Y ojalá que no sea tan tarde, ¿verdad? Que se dé cuenta pronto la humanidad de qué está pasando y por qué está pasando. Por qué son los problemas, las enfermedades [...] Y ojalá ayude un poco el gobierno y se enfocara más en lo que estamos trabajando aquí, los que estamos tratando de trabajar naturalmente [...] Aquí no ocupamos nada. Sí ocupamos apoyo y todo, pero si queremos, nosotros podemos depender de nosotros mismos. Nomás es de olvidarnos de cosas que realmente no son necesarias pero que realmente las tenemos.

Todos los efectos que reconocen estos campesinos: la tierra cansada y erosionada, la pérdida de materia orgánica en el suelo, las enfermedades, la resistencia de malezas y debilidades en las plantas tradicionales, son señales de que hay necesidad de un cambio. Como dijo Moncho: "Ojalá que no sea tan tarde. La tierra hay que sustentarla y conservarla, porque de ella sale el sustento de la gente y porque en ella está tejida la cultura de los mexicanos". 


\section{Discusión y conclusión}

Al discutir los resultados de esta investigación en el contexto del desarrollo sustentable, es importante reconocer que la sustentabilidad es un fenómeno multidimensional, es decir, tiene implicaciones ecológicas, económicas y sociales altamente interrelacionadas; multiactor, con la presencia de diversos actores sociales; y multinivel, que tiene interrelaciones entre lo local y lo global, en vez de hablar de un concepto fragmentado, aislado y limitado a un solo actor social (Gerritsen, 2006). Asimismo, usamos la división de productores orgánicos y convencionales para comparar percepciones, aunque reconocemos que estos cuatro campesinos también tienen muchas cosas en común. Todos comparten la meta principal de sobrevivir como productores con recursos limitados y buscar sustento para sus familias. La base de este trabajo es rescatar las palabras de cada uno, enfocándonos en el valor de sus conocimientos individuales, o como lo dice el pedagogo brasileño Paulo Freire: "El conocimiento se construye a partir del diálogo entre los seres humanos en torno a su mundo, a su realidad; de este modo todos somos sabios y todos somos ignorantes, respecto a diferentes dimensiones de la realidad, lo que sucede es que hemos bebido de distintas fuentes del conocimiento" (1985: 3). En otras palabras, encontramos conocimiento en el diálogo que se genera cuando escuchamos y valoramos la realidad de cada persona.

En esta última sección del artículo hacemos un esfuerzo integrador de las percepciones y experiencias presentadas, y analizamos las implicaciones de las mismas para el diseño e implementación de políticas públicas y esquemas de desarrollo sustentable. A continuación nos enfocamos primero en las diferencias entre los cuatro campesinos, para posteriormente describir las semejanzas. Terminamos con algunas reflexiones en torno a las políticas públicas que buscan impulsar el desarrollo sustentable.

\section{Diferencias entre los cuatro productores}

Es evidente que existe una diferencia importante en cuanto al grado de dependencia entre los agricultores convencionales y los orgánicos. El trabajo de los productores convencionales se refleja en un fuerte vínculo con factores externos: en los insumos para la producción, en la sujeción de la demanda del mercado, en los intermediarios y en la ideología impuesta por la modernidad. Esta ideología despojó el destino de su producción: del autoconsumo a 
la venta. En cambio, los campesinos orgánicos aprovechan los recursos locales para crear opciones de producción menos dependiente: usan herramientas de antaño; utilizan su propia semilla; producen para autoabastecerse y alimentar a sus animales, además de vender sus excedentes en la región, lo que refuerza la seguridad alimentaría de sus familias y su comunidad. Un tema fuerte en su discusión es tener poder, tomar de decisiones y usar su creatividad para buscar alternativas con poco impacto ambiental y menor gasto económico. En diversas ocasiones enfatizaron la importancia de actuar con autonomía, buscando sus propias maneras de sobrevivir con lo que puedan trabajar con sus propias manos, producir con los recursos de la comunidad y experimentar poniendo en práctica sus conocimientos.

En la descripción del trabajo en su parcela, descubrimos que las prácticas de los campesinos convencionales son menos complejas puesto que involucran un número menor de pasos en el ciclo de producción. Por el lado de los agricultores orgánicos, nos comentaron sobre un trabajo más íntegro y complejo, además destacaron su orgullo respecto de una relación más intensa con la tierra. En varias ocasiones mencionaron su gusto por trabajar con fuerza para lograr sus metas. Nos platicaron detalladamente y con afectividad sobre este tema, mientras que a los productores convencionales no les animó tanto. En comparación, uno de los productores convencionales, José, comentó que por la intervención de maquinaria, se ha vuelto perezoso para trabajar y cuidar su terreno lo mejor posible. Aquí se refleja el gusto de los agricultores orgánicos por trabajar la tierra de manera esmerada, cosa que no ocurrió con los convencionales.

$\mathrm{Al}$ analizar las entrevistas, se evidenció que los cuatro campesinos perciben algún efecto de la degradación ambiental en sus vidas, como ya mencionamos, aunque sólo tres respondieron así en la pregunta directa correspondiente. Mencionaron efectos en la salud, en el bienestar familiar y en factores económicos. Cabe destacar que los productores orgánicos mostraron efectos más sentimentales mientras los convencionales enfatizaron la cuestión económica. Las respuestas más extremas vinieron de Osberto, productor convencional, y Raúl, productor orgánico. Osberto respondió que no percibía un efecto personal con respecto a la degradación ambiental (aunque en varias otras partes de la entrevista mencionó los espectos económicos). En cambio, Raúl contestó que tiene un efecto directo en su bienestar emocional, espiritual y mental. 
En general, los seres humanos tenemos una frágil relación con la tierra, pero los campesinos, que son quienes interactúan más directamente con ella, trabajándola, desbordan su pasión y devoción por hacerla producir de la mejor manera de acuerdo con su criterio. En este sentido, encontramos una diferencia notable entre los campesinos convencionales y los orgánicos. Los primeros mencionaron una relación de subsistencia y sustento para ellos y sus familias. Los segundos revelaron que no sólo obtienen su mantenimiento físico de ella, también todos los aspectos diferentes de sus vidas, mostrando una relación bastante profunda y enraizada con la tierra. En agradecimiento a esto la alaban, la sobrevalúan, le dan cariño, alimento, amor, cuidado y un aprecio extraordinario. De esta manera no sólo la trabajan, sino que conviven con ella como madre y a cada uno de sus elementos y características le atribuye toda la importancia necesaria para mantener su existencia por siempre. A través de sus perspectivas, notamos de los campesinos orgánicos por cultivar usando estos métodos viene de un paradigma de creencias profundas, espiritualidad, agradecimiento y valor hacia la tierra (Toledo, 2000, 2003; Foster 1960; Van Eijk, 1998). Ven en la agricultura la importancia de la continuación de tradiciones y valores antiguos y conciben la naturaleza como una parte fundamental de la vida del ser humano, que viene de un pensamiento integrador holístico (Mendras, 1970; Toledo, 2000). Sin embargo, la modernización de la agricultura ha llegado al grado de desarticular la valoración de la naturaleza como un todo y disociar las interacciones entre los elementos del sistema del pensamiento del agricultor (Van der Ploeg, 1987, 1992, 1999; Gerritsen, 2002; Guzmán et al. 2000; Morales, 2004).

Las diferencias entre los cuatro productores estudiados se resumen en las tablas 1 a 4 mediante un número limitado de criterios o indicadores (cualitativos) para la sustentabilidad, ubicados en el ámbito ecológico, social, económico e institucional (Morales, 2003). Cabe mencionar que la calificación en las tablas es relativa y tiene validez más que nada en la comparación de los cuatro productores.

\section{Semejanzas entre los cuatro productores}

Para empezar, uno de los principales problemas en que los cuatro campesinos concordaron es la tecnología que trajo la Revolución Verde, misma que les acarreó consecuencias negativas como pequeños propietarios, y que se manifiestan en muchos 
Tabla I

Diferencias ecológicas entre los cuatro productores

\begin{tabular}{|c|c|c|c|c|}
\hline Indicadores & Moncho & Raúl & Osberto & José \\
\hline $\begin{array}{l}\text { Incorporación de materia } \\
\text { orgánica }\end{array}$ & Mucha & Mucha & Poca & Media \\
\hline Aplicación de pesticidas & Nula & Nula & Media & Media \\
\hline Rotación de cultivos & Cada ciclo & Cada ciclo & Nunca & Poca \\
\hline Uso de semilla local & Siempre & Siempre & Nunca & Nunca \\
\hline $\begin{array}{l}\text { Asociación de cultivos y } \\
\text { cercos vivos }\end{array}$ & Mucha & Mucha & Poca & Media \\
\hline
\end{tabular}

Tabla II

Diferencias sociales entre los cuatro productores

\begin{tabular}{|c|c|c|c|c|}
\hline Indicadores & Moncho & Raúl & Osberto & José \\
\hline Mano de obra familiar & Baja & Alta & Nula & Nula \\
\hline Apego hacia la tierra & Estrecha & Estrecha & Baja & Media \\
\hline $\begin{array}{l}\text { Conocimiento de los ciclos } \\
\text { naturales de su entorno }\end{array}$ & Alto & Alto & Bajo & Medio \\
\hline $\begin{array}{l}\text { Grado de participación en } \\
\text { la comunidad }\end{array}$ & Medio & Alto & Bajo & Medio \\
\hline Transmisión de saberes & Alta & Alta & Bajo & Bajo \\
\hline
\end{tabular}

Tabla III

Diferencias económicas entre los cuatro productores

\begin{tabular}{lllll}
\hline \multicolumn{1}{c}{ Indicadores } & Moncho & Raúl & \multicolumn{1}{c}{ Osberto } & José \\
\hline Ahorro & Mucho & Mucho & Nulo & Nulo \\
Autosuficiencia & Mucha & Mucha & Muy poca & Poca \\
Inversión en la producción & Baja & Baja & Media & Alta \\
Permite varias cosechas a la vez & Muchas & Muchas & Una & Pocas \\
Grado de endeudamiento & Nulo & Nulo & Alto & Alto \\
\hline
\end{tabular}

Tabla IV

Diferencias institucionales entre los cuatro productores

\begin{tabular}{lllll}
\hline \multicolumn{1}{c}{ Indicadores } & Moncho & Raúl & Osberto & José \\
\hline Subsidios del gobierno & Poco & Poco & Nulo & Poco \\
Asesoría externa & Nula & Nula & Poca & Poca \\
Inversión externa & Nula & Nula & Nula & Nula \\
Adopción de tecnologías & Nula & Nula & Media & Media \\
Dependencia del mercado & Nula & Nula & Alta & Alta \\
\hline
\end{tabular}


aspectos. Para seguir con el método convencional tienen que invertir más en insumos de maquinaria y agroquímicos, que en general no compensa el rendimiento y valor del producto en el mercado. Como otro efecto mencionaron la fragmentación de la cultura campesina, lo cual se evidencia en la pérdida de técnicas tradicionales, de comunicación y organización entre los campesinos, de cultivos tradicionales, de semillas criollas, de la ética de trabajo, de soberanía y autonomía, de conocimientos sobre los procesos naturales y los lazos de relación íntima entre el campesino y la tierra.

Tanto los agricultores orgánicos como los convencionales percibieron una degradación ambiental en su entorno, la cual enfatizaron de diferentes maneras: degradación del suelo, intoxicación y tala de árboles, disminución de la fertilidad y contaminación de cultivos tradicionales. En general todos coincidieron en que la forma actual de trabajar no está dando los resultados esperados, por lo que plantearon la búsqueda de opciones para mejorar la situación. En palabras de Morales, "el camino hacia el desarrollo sustentable se origina a partir de diferentes estrategias, orientadas hacia la búsqueda de alternativas al desarrollo modernadizador" (2003: 19; ver también Van der Ploeg, 1994; Gerritsen, 2002). Moncho, Raúl y José hablaron de buscar una solución más de fondo en cuanto a la salud de la tierra, mediante un esquema cuya base sea una agricultura sana y productiva. Por su parte, Osberto propuso sustituir los cultivos por otros más resistentes y con menor demanda de nutrientes, una solución que no va a la raíz del problema, pero también busca una alternativa.

\section{Algunas reflexiones sobre percepciones campesinas $y$ políticas públicas}

A lo largo de este trabajo notamos que los productores entrevistados, cada uno desde su perspectiva, coinciden en que la principal limitación para desarrollar su trabajo y obtener mejores resultados está en el modelo neoliberal de desarrollo, el que, de acuerdo con Morales, "Se basa en el mercado como el eje de la industrialización de la agricultura, y excluye las consideraciones sociales, ecológicas y culturales, en razón del crecimiento económico" (2003: 30). Algunas limitaciones de este sistema son la falta de apoyo del gobierno, los precios del mercado y las políticas agropecuarias inadecuadas. Por esta razón, cada uno, de acuerdo con sus impedimentos, crea ciertas resistencias (Scott, 1985). Con ello intentan asegurar la permanencia continua de su siste- 
ma de producción, de su identidad cultural y de su posibilidad de seguir existiendo como comunidad rural. Tanto los productores orgánicos como los convencionales ven como una esperanza que el gobierno reconsidere su postura ante el campo, por medio de un mayor subsidio a la producción agropecuaria y el desarrollo de programas encaminados a la producción orgánica o, por lo menos, que promuevan opciones más naturales y accesibles para la economía del campesino (Swentesius et al., 2003).

En este sentido, es ofensivo y humillante que el gobierno no haya puesto atención en el campo para escuchar las demandas campesinas (ibidem). Los agricultores necesitan un modelo de desarrollo encaminado desde las perspectivas local y de sustentabilidad. Ante esta desatención, han iniciado la búsqueda de opciones independientemente. En esta investigación encontramos que los productores orgánicos desean adentrarse más en su trabajo y hacerse más autónomos y autosuficientes. Por su parte, los agricultores convencionales tratan de iniciar una transición hacia lo orgánico y averiguar qué cultivos requieren menos nutrientes y son más resistentes a las plagas, para introducirlos a su sistema de producción. Ya sea mediante la creación de nuevas técnicas de agricultura, el avance y aumento del trabajo en agricultura orgánica, de la diversificación de actividades productivas, la introducción de nuevos cultivos, la inserción en movimientos sociales de lucha por la dignidad y resistencia a la crisis participando y organizándose mejor en su comunidad, todos los campesinos que entrevistamos coinciden en que el modelo actual de desarrollo, con sus técnicas impuestas, está exterminando la tierra, su vida y con ello el trabajo campesino, la sabiduría ancestral y las culturas que han forjado a México con el paso de los años.

Por todo lo anterior creemos que para, de alguna manera, salir de la crisis actual del campo mexicano, es necesario elaborar proyectos desde la base cuyo impulso primordial son las percepciones y la ideología del campesino (Toledo, 2000; Morales, 2004; Guzmán et al., 2000; Villalvazo et al., 2003; Gerritsen, 2002).

Con esto último nos referimos a que las percepciones son un reflejo de las estrategias productivas de los campesinos, así como de las condiciones locales (de índole ecológico, económico, institucional y social) en que se desarrollan. El partir de las percepciones, estrategias productivas y condiciones locales, requiere retomar la comunidad o la región como eje central de la planeación gubernamental, eje que fue abandonado a principios de los años ochenta del siglo pasado, con la introducción de las ideas neoliberales en la políticas nacionales (Ferreira, 2005). En otras 
palabras, el diseño e implementación de enfoques regionales implica que más que basar las políticas en supuestos que se definen en ámbitos nacionales e internacionales, se tiene que partir de lo local, es decir, de las comunidades indígenas y ejidos mexicanos. Para lograr esto, se debe tener una comunicación efectiva entre el Estado y los productores para que éstos puedan comunicar sus percepciones, las cuales deben ser clave en los discursos gubernamentales (Toledo y Bartra, 2000).

El estudio que realizamos es una muestra del valor de la sabiduría del campesino y de cómo la riqueza de sus palabras y pensamientos puede generar ideas piloto que creen opciones. Mientras esto siga excluido, la situación de las zonas rurales empeorará y su riqueza cultural desaparecerá. La modernización y artificialización de la agricultura no toma en cuenta el pensar y sentir del agricultor ni sus condiciones específicas. Se olvida de que sus percepciones y pensamientos manifiestan y resaltan su voz en el trabajo que ha sido capaz de sustentar a la humanidad por miles de años (Diamond 1999; Arrighi, 1999). El punto de partida para discutir soluciones en los ámbitos comunitario, académico o gubernamental debe empezar con solicitar y escuchar estas voces campesinas.

\section{Bibliografía}

Arnon, Inbal (1987), La modernización de la agricultura en países en vía de desarrollo: recursos, potenciales, problemas, México, Limusa.

Arrighi, Giovanni (1999), El largo siglo xx. Dinero y poder en los orígenes de nuestra época, Madrid, Akal.

Bonfil, Guillermo (1994), México profundo: una civilización negada, México, Grijalbo.

Diamond, Jared (1999), Guns, germs and steel. The fates of human societies, Nueva York-Londres, W. W. Norton.

Eijk, Toon Van (1998), Farming systems research and spirituality. An analysis of the foundations of professionalism in developing sustainable farming systems, tesis de doctorado, Wageningen, Países Bajos, Universidad de Wageningen. 
Ferreira, Héctor (2005), Construir las regiones. Por una aproximación regional a la formulación y gestión de politicas publicas en México, México, Centro Lindavista-Integración Editorial.

Forster, George (1960), Culture and conquest. America's Spanish heritage, Chicago, Quadrangle Books, Viking Fund Publications in Anthropology.

Freire, Paulo (1985), La política de educación: cultura, poder y liberación, South Hadley, Massachussets, Bergin \& Garvey.

Gerritsen, Peter (2006), "Globalización y desarrollo regional sustentable. Algunas reflexiones teóricas”, ponencia presentada en la XVII Semana Nacional de la Investigación Científica, con subsede en el Centro Universitario de la Costa Sur de la Universidad de Guadalajara, Autlán, Jal., 22-30 de noviembre de 2006.

Gerritsen, Peter (2002), Diversity at stake. A farmer's perspective on biodiversity and conservation in western Mexico, Wageningen University.

Gerritsen, Peter, Alma Lomelí y Claudia Ortiz (2005), "Urbanización y problemática socioambiental en la Costa Sur de Jalisco, México: una aproximación”, Región y Sociedad, XVII(33), pp. 107-132.

Gliessman, Stephen (2000), Agroecology: Ecological Processes in Sustainable Agriculture, Boca Raton, Florida, Lewis Publishers.

Gónzalez-Figueroa, Rodolfo (2006), Análisis comparativo de cuatro cultivos en base a principios de sustentabilidad, en La Ciénega, municipio de El Limón, tesis de licenciatura, México, Centro Universitario de la Costa Sur, Universidad de Guadalajara.

Guzmán, Gloria, Manuel González de Molina y Eduardo Sevilla Guzmán (2000), Introducción a la agroecología como desarrollo rural sostenible, Madrid-Barcelona-México, Mundi-Prensa. 
Hurni, Hans, Urs Wiesman y Roland Schertenleib (2004), Research for mitigating syndromes of global change. A transdisciplinary appraisal of selected regions of the world to prepare development oriented research partnerships, Berna, Universidad de Berna. Geographica Bernensia, Perspectives of the Swiss National Centre of Competence in Research (NCCR) North-South, 1.

Imecbio (Instituto Manantlán de Ecología y Conservación de la Biodiversidad) (2000), Programa de desarrollo regional sustentable, nivel regional, México, Universidad de Guadalajara.

Jansen, Kees y Sietze Vellema (2004), Agribusiness and Society, Corporate Responses to Environmentalism, Market Opportunities and Public Regulation, Londres-Nueva York, Zed Books.

Lazos, Elena y Luisa Paré (2000), Miradas indígenas sobre una naturaleza entristecida. Percepciones del deterioro ambiental entre nahuas del sur de Veracruz, México, IIs-Universidad Nacional Autónoma de México-Plaza y Valdés.

Long, Norman y Ann Long (eds.) (1992), Battlefields of knowledge. The interlocking of theory and practice in social research and development, Londres-Nueva York, Routledge.

Long, Norman (2001), Development sociology. Actor perspectives, Londres-Nueva York, Routledge.

Long, Norman y Jan Douwe van der Ploeg (1989), "Demythologizing planned intervention: an actor perspective", Sociología Rurales, xxix(3/4), Wageningen (Países Bajos), pp. 226-249.

Long, Norman y Jan Douwe van der Ploeg (1994), "Hetereogeneity, actor and structure: towards a reconstitution of the concept of structure", en David Booth (ed.), Rethinking social development: theory, research and practice, Essex, Longman Scientific and Technical, pp. 62-89.

Mendras, Henry (1970), The vanishing peasant: innovation and change in French agriculture, Cambridge, Cambridge University Press. 
Morales, Jaime (2003), Desarrollo rural alternativo en el sur de Jalisco: experiencias hacia la sustentabilidad rural, informe final de investigación, Instituto Tecnológico y de Estudios Superiores, Guadalajara.

Morales, Jaime (2004), Sociedades rurales y naturaleza. En busca de alternativas hacia la sustentablidad, Tlaquepaque-Guanajuato, Instituto Tecnológico y de Estudios SuperioresUniversidad Iberoamericana.

Ploeg, Jan Douwe van der (1987), De verwetenschappelijking van de landbouwbeoefening, Wageningen, Países Bajos, Wageningen Agricultural University.

Ploeg, Jan Douwe van der (1992), "The reconstitution of locality: technology and labour in modern agriculture", en Tierry Marsden, Rob Lowe y Sarah Whatmore (eds.), Labour and locality: uneven development and the rural labour process, Londres, David Fulton Publishers, pp. 19-43.

Ploeg, Jan Douwe van der (1999), De virtuele boer, Assen, Países Bajos, Van Gorcum Publishers.

Scott, James (1985), Weapons of the weak. Everyday forms of peasant resistance, New Haven-Londres, Yale University Press.

Swentesius, Rita, Manuel Gómez, José Luis Calva y Luis Hernández (coords.) (2003), ¿El campo aguanta más, México, Universidad Autónoma de Chapingo, CIESTAM.

Toledo, Víctor (2000), La paz en Chiapas. Ecología, luchas indigenas y modernidad alternativa, México, Ediciones Quinto Sol-Universidad Nacional Autónoma de México.

Toledo, Víctor (2003), Ecología, espiritualidad y conocimiento. De la sociedad de riesgo a la sociedad sustentable, México, Universidad Iberoamericana-Programa de las Naciones Unidas para el Medio Ambiente.

Toledo, C. y A. Bartra (coords.) (2000), Del círculo vicioso al círculo virtuoso. Cinco miradas al desarrollo sustentable de las regiones marginadas, México, Semarnat. 
Villalvazo, Víctor, Peter Gerritsen, Pedro Figueroa Bautista y Gerardo Cruz Sandoval (2003), "Desarrollo rural endógeno en la Reserva de la Biosfera Sierra de Manantlán”, Sociedades Rurales. Producción y Medio Ambiente 4(1), México, pp. 41-50.

Recibido: 1 de septiembre de 2006. Reenviado: 4 de diciembre de 2006. Aceptado: 19 de enero de 2007.

Rodolfo González Figueroa. Es ingeniero en recursos naturales y agropecuarios, Departamento de Ecología y Recursos Naturales-Imecbio, Centro Universitario de la Costa Sur, Universidad de Guadalajara. Sus líneas actuales de investigación son: modos de producción, agricultura tradicional, uso y manejo de los recursos naturales. Ha publicado: Análisis comparativo de cuatro cultivos en base a principios de sustentabilidad en el ejido de La Ciénega, Mpio. de El Limón, Jalisco, Autlán, Universidad de Guadalajara, Centro Universitario de la Costa Sur.

Peter R. W. Gerritsen. Es doctor en Ciencias sociales, Departamento de Ecología y Recursos Naturales-Imecbio, Centro Universitario de la Costa Sur, Universidad de Guadalajara. Líneas actuales de investigación: percepción, uso y manejo campesino de recursos naturales; tenencia y manejo de recursos naturales; género y manejo de recursos naturales; globalización, urbanización y manejo de recursos naturales. Es miembro del Sistema Nacional de Investigadores. Ha publicado: y K.F. Wiersum (2005). "Farmer and conventional perspectives on conservation in western Mexico", Mountain Research and Development, 25(1), 2005, 30-36; Estilos agrarios y la forestería comunitaria. Estudio de caso de la comunidad indígena de Cuzalapa en la reserva de la biosfera sierra de Manantlán en el Occidente de México, México, Universidad de Guadalajara, Centro Universitario de la Costa Sur (2004); Barrera, A., J.C. Bolay, C. García, S. Hostettler en colaboración con P.R.W. Gerritsen, R. Mejía, C. Ortiz, M. Sánchez, Y. Pedrazzinni, L. Poschet y A. Rabinovich (2004). 'JACS Central America and the Caribbean. Key challenges of sustainable development and research priorities: social practices as driving forces for change. Pp. 293-327 en Hurni, H., U. Wiesman y 
R. Schertenleib (2004). Research for mitigating syndromes of global change. A transdisciplinary appraisal of selected regions of the world to prepare development oriented research partnerships Berne: University of Berne: Geographica Bernensia. Perspectives of the Swiss National Centre of Competence in Research (NCCR) North-South, Vol. 1.; Diversity at stake. A farmers' perspective on biodiversity and conservation in Western Mexico: Wageningen, Países Bajos, Wageningen University, The Netherlands. Wageningen Studies on Heterogeneity and Relocalisation.

Therese K. Malischke. Es licenciada en sociología rural y estudios latinoamericanos, caribeños e ibéricos. Universidad de Wisconsin-Madison. Líneas actuales de investigación: jardines comunitarios. 